\title{
Scaling laws of simulated galaxy clusters
}

\author{
Debora Sijacki ${ }^{1,2}$ and Giuseppe Tormen ${ }^{2}$ \\ ${ }^{1}$ MPA, Garching, Germany email: deboras@mpa-garching.mpg.de \\ ${ }^{2}$ Dipartimento di Astronomia, Università di Padova, Italy email: tormen@pd.astro.it
}

\section{Introduction}

Using high-resolution non radiative SPH simulations we study the scaling laws of galaxy clusters. In particular, we analyze the relations existing between total mass, gas temperature, X-ray luminosity and dark matter velocity dispersion. Our aim is to understand how these scaling laws depend on numerical, statistical and physical parameters, in order to model and thus reduce potential systematical uncertainties. The simulations were performed by the GADGET code in a $\Lambda C D M$ cosmology, applying resimulation techniques; their mass resolution ranges between $2 \times 10^{9}$ and $6.8 \times 10^{9} h^{-1} M_{\odot}$ for dark matter particles and between $3 \times 10^{8}$ and $7 \times 10^{8} h^{-1} M_{\odot}$ for gas particles.

\section{Method}

We evaluated the scaling relations on a regular grid of radii (from $0.05 R_{v i r}$ to $5 R_{v i r}$ with step of $0.02 R_{\text {vir }}$ ) and overdensities (from $2 \cdot 10^{4} \rho_{c}$ to $30 \rho_{c}$ with an adaptive step) $†$. Scaling laws were determined at each radius (or overdensity) using cumulative physical quantities corresponding to that radius (or overdensity) and were compared with the isothermal model (see Figs. 2 and 3). The redshift evolution of scaling relations was evaluated by taking the five snapshots closest to $z=0,0.25,0.5,0.75$ and 1 . We investigated how the zero point and slope of the scaling laws depend on the number of dark matter particles used to describe a halo (see Fig. 1). We conclude that the value $N_{\text {lim }}$ is a good compromise between numerical effects (which influence more strongly systems with less than 1000-2000 DM particles), convergence criteria (scaling laws stabilize conservatively above $N_{\text {lim }}$ ) and dynamical evolution (more massive systems are more likely to be less dynamically relaxed and thus may introduce a bias in the evaluation of scaling laws).

\section{Properties at redshift zero}

The slope of the $M(T)$ relation in the inner regions $\left(<0.2 R_{v i r}\right)$ is consistent with the self-similar prediction, while moving to larger radii it increases. This happens because at the large radii the energy budget has a non negligible contribution from the gas bulk motions (consistent with the $\beta$ parameter of $\sim 1.17$ at $R_{v i r}$ ), causing a temperature decrease. Variation with radius in the $L_{X}(T)$ and $L_{X}(M)$ relations are less evident. These two relations also require a higher number of objects to be accurately defined and to reduce the scatter. The slope of the $L_{X}(T)$ relation is to very good approximation consistent with the theoretically predicted value at all radii, while the slope of the $L_{X}(M)$ relation is somewhat underestimated. This results may reflect the fact that the incomplete gas thermalization affects the evaluation of $L_{X}$. The slope of the $M\left(\sigma_{D M}\right)$ relation is close

$\dagger$ Especially considering the redshift evolution of scaling laws these two approaches can lead to different results, due to their correspondence to different regions of clusters. 

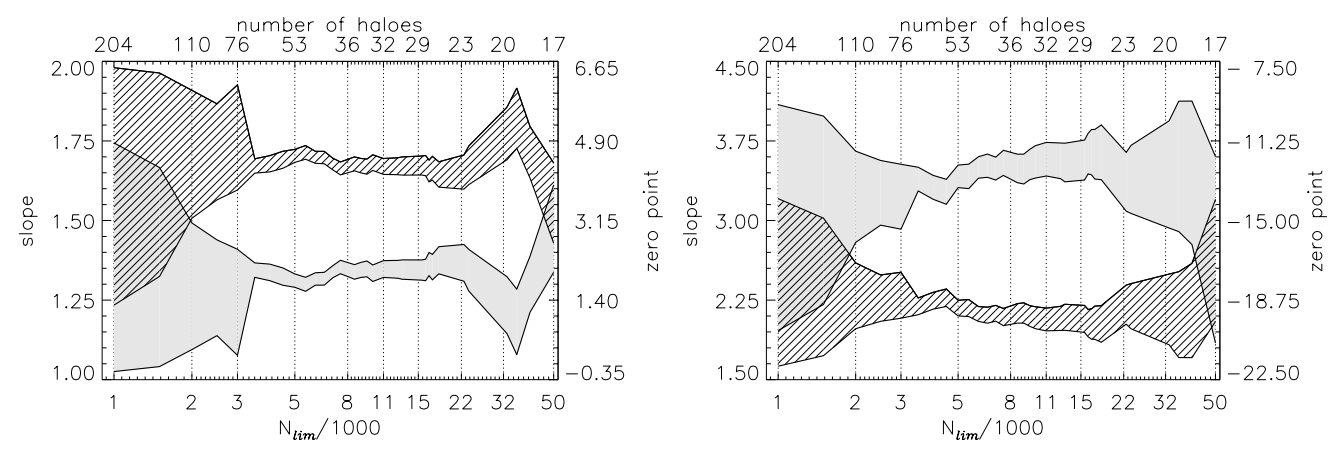

Figure 1. Left panel: $M_{v i r}(T)$ scaling law, right panel: $L_{X}(T)$ relation. Hatched bands correspond to the slope of the relation (scaling law + its inverse) and the gray bands to the zero point. The bottom $\mathrm{x}$-axis indicates the threshold in the number of dark matter particles on a logarithmic scale and the upper one shows the number of galaxy clusters having at least that threshold number.
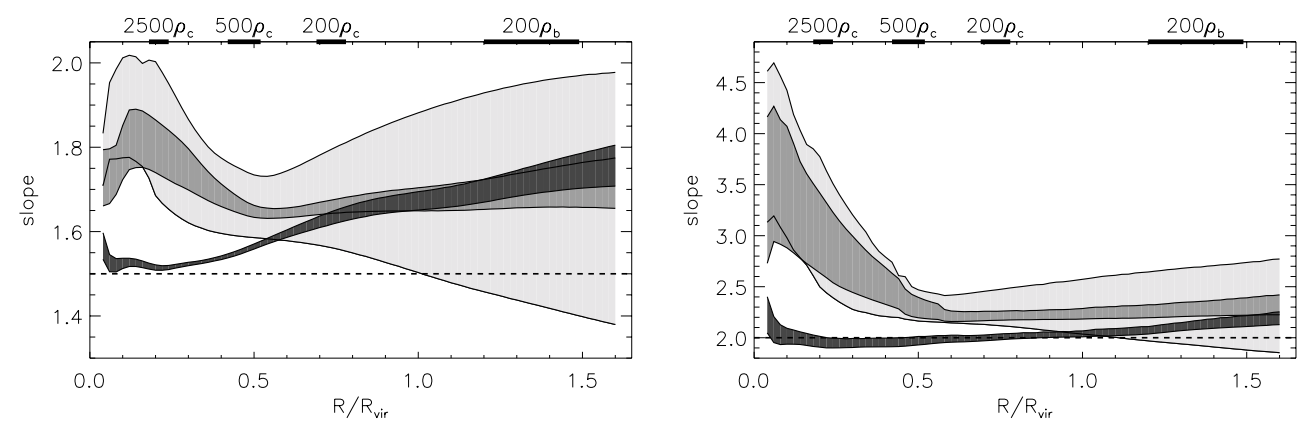

Figure 2. Slope of the $M(T)$ and $L_{X}(T)$ scaling laws evaluated on a regular grid of radii for 3 choices of $N_{\text {lim }}=2000,4000$ and 8000 (from light to dark gray respectively), for which the scatter is substantially reduced. The continuous line gives the theoretically predicted value.

to the theoretical prediction at large radii, while in the inner regions it is systematically lower. This is due to the mass dependence of the surface pressure term present in the generalized virial theorem equation. The $\sigma_{D M}(T)$ scaling law shows in a direct way effects of non complete thermalization, which are more prominent in outer regions and in massive clusters.

\section{Redshift evolution of scaling laws}

The different scaling laws exhibit a redshift dependence that can be expressed by power laws of $H(z)$ and $\Delta_{c}$. If we factor this out, no evolution should be observed. To first approximation, this is true also for the relations followed by our simulated clusters. Nevertheless, some systematical trends remain (see Fig. 4). In the case of the $M(T)$ and $M\left(\sigma_{D M}\right)$ scaling laws, in the inner clusters regions, for a given $T$ (or $\sigma_{D M}$ ) the total mass inside the fixed radius decreases with increasing redshift. This can be understood as follows: the mass profile in the inner regions is more affected by the value of the concentration parameter, and the mean concentration of our cluster sample at $z=1$ is $\sim 4.9$, while at $z=0$ it increases to $\sim 8.1$. The $\sigma_{D M}(T)$ relation follows the selfsimilar evolutionary scenario in the inner regions, while at larger radii there is evidence that the temperature systematically increases with time. This can be also seen for the 

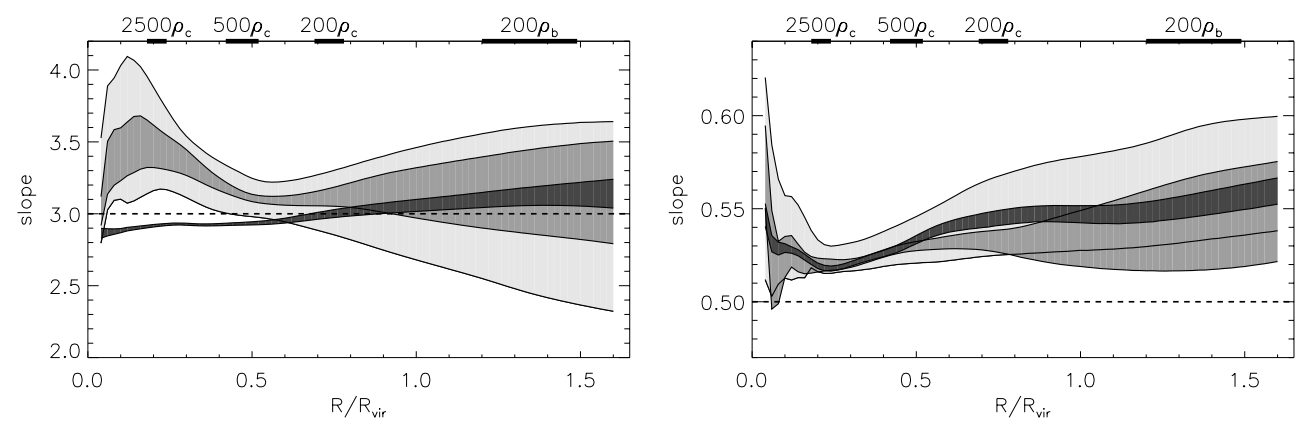

Figure 3. Slope of the $M\left(\sigma_{D M}\right)$ and $\sigma_{D M}(T)$ relations. Same description as in the previous figure.
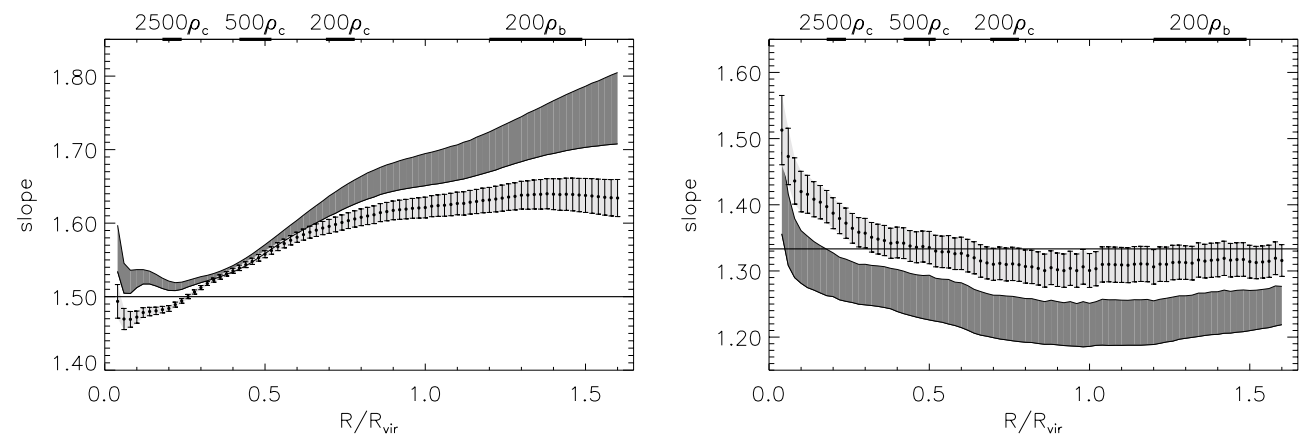

Figure 4. Slope of the $M(T)$ and $L_{X}(M)$ scaling laws evaluated on a regular grid of radii at redshift zero (gray band) and at 5 redshifts taken together (band with error bars).

other scaling laws involving $T$, and the same can be concluded regarding $L_{X}$. In order to verify if these trends can be explained by the redshift evolution of the concentration, we modeled it (as predicted by Eke et al. 1998) obtaining substantial improvement and better agreement with the self-similar evolutionary picture.

\section{Conclusions}

Generally speaking, substructures affect cluster properties in a more prominent way at larger radii, causing a bigger scatter in the estimated quantities. On the other hand, the mass enclosed in a given radius depends on the concentration parameter mostly in the inner regions, and this leads to systematic biases in the scaling laws compared to the isothermal prediction. Moreover, the redshift evolution of halo concentrations leads to further disagreement with respect to the self-similar picture. Once clusters are resolved with sufficient numerical accuracy, the scaling laws are more tightly defined and are less influenced by the cluster dynamical state in the inner regions. Finally, it is interesting to note that deviations from self-similarity appear in the scaling relations also in adiabatic hydrodynamics: this may suggest that a more accurate physical model of scaling laws, that models in a realistic manner profiles of various physical quantities in question, should be used. 


\section{References}

Borgani, S., Murante, G., Springel, V., Diaferio, A., Dolag, K., Moscardini, L., Tormen, G., Tornatore, L. and Tozzi, P. 2004 MNRAS 348, 1078-1096.

Bryan, G. L. and Norman, M. L. 1998 ApJ 495, 80

Eke, V. R., Navarro, J. F., and Frenk, C. S. 1998 ApJ 503, 569

Evrard, A. E., Metzler, C. A., and Navarro, J. F. 1996 ApJ 469, 494

Kuhlen, M., Strigari, L. E., Zentner, A. R., Bullock, J. S., and Primack, J. R. 2004 draft version (preprint astro-ph/0402210)

Navarro, J. F., Frenk, C. S., and White, S. D. M. 1995 MNRAS 275, 720-740.

Rasia, E., Tormen, G. and Moscardini, L. 2004 MNRAS, in press (preprint astro-ph/0309405)

Tormen, G., Moscardini, L., and Yoshida, N. 2003 MNRAS, submitted (preprint astro$p h / 0304375)$ 